\title{
Vortex and soliton dynamics in particle-hole-symmetric superfluids
}

\author{
Jim Skulte $\odot,{ }^{1,2}$ Lukas Broers $\odot,{ }^{1}$ Jayson G. Cosme $\odot,{ }^{3}$ and Ludwig Mathey ${ }^{1,2}$ \\ ${ }^{1}$ Zentrum für Optische Quantentechnologien and Institut für Laserphysik, Universität Hamburg, 22761 Hamburg, Germany \\ ${ }^{2}$ The Hamburg Centre for Ultrafast Imaging, Luruper Chaussee 149, 22761 Hamburg, Germany \\ ${ }^{3}$ National Institute of Physics, University of the Philippines, Diliman, Quezon City 1101, Philippines
}

(Received 15 July 2021; accepted 21 October 2021; published 12 November 2021)

\begin{abstract}
We propose to induce topological defects in particle-hole-symmetric superfluids, with the prime example of the Bardeen-Cooper-Schrieffer state of ultracold atoms, and detect their time evolution and decay. We demonstrate that the time evolution is qualitatively distinct for particle-hole-symmetric superfluids and point out that the dynamics of topological defects is strongly modified in particle-hole-symmetric fluids. We obtain results for different charges and compare them with the standard Gross-Pitaevskii prediction for Bose-Einstein condensates. We highlight the observable signatures of the particle-hole symmetry in the dynamics of decaying solitons and subsequent vortices.
\end{abstract}

DOI: 10.1103/PhysRevResearch.3.043109

\section{INTRODUCTION}

The presence or absence of particle-hole symmetry in a physical system is a fundamental property pervading its dynamical properties. Particle-hole symmetry is realized in Lorentz invariant theories such as the standard model of elementary physics [1], low-energy effective models close to quantum criticality [2], and the famous Bardeen-CooperSchrieffer (BCS) theory of superconductivity [3,4]. ${ }^{1}$ We note that the order parameter dynamics of high- $T_{\mathrm{c}}$ superconductors can be described by an effective particle-hole-symmetric theory, which allows for exploring the dynamics of the Higgs/amplitude mode [5-8]. Similarly, in ultracold neutral atoms the emergence of an effective particle-hole symmetry has been predicted theoretically $[9,10]$ and confirmed experimentally $[11,12]$. Recently, amplitude oscillations of the order parameter in the Bose-Einstein condensate (BEC) to BCS crossover have been reported [13], suggesting the presence of approximate particle-hole symmetry.

The dynamics of topological defects, such as solitons and quantized vortices, derives from and exemplifies the properties of the underlying quantum fluid. The stability of solitons has been discussed extensively for the nonlinear Schrödinger equation or Gross-Pitaevskii (GP) equation [14-18]. Zakharov and Rubenchik coined the term snaking to refer to the characteristic bending of solitons prior to their decay.

\footnotetext{
${ }^{1}$ Due to the close connection between relativistic Lorentz invariance and particle-hole symmetry, models that are particle-hole symmetric are also sometimes referred to as relativistic models.

Published by the American Physical Society under the terms of the Creative Commons Attribution 4.0 International license. Further distribution of this work must maintain attribution to the author(s) and the published article's title, journal citation, and DOI.
}

Snaking is a manifestation of the Magnus force. This has been discussed for neutral bosonic systems within the GP equation [19-25], in the BEC-BCS crossover [26-28], and in superconductors $[29,30]$.

We propose to determine the influence of particle-hole symmetry on the dynamics of topological defects in twodimensional neutral superfluids. We focus on the BCS state as our primary example, but our results hold for any approximately particle-hole-symmetric system, e.g., bosons in an optical lattice near unit filling [12]. For this purpose we present the similarities and differences in the dynamics of topological defects in the absence and presence of particle-hole symmetry. We also compare the dynamics of the particle-hole-symmetric theory for zero and nonzero Noether charge, corresponding to a balanced mixture of particles and holes and an imbalanced mixture of particles and holes, respectively. We find that the case with nonzero charge is reminiscent of the dynamics of the GP equation. On the other hand, for vanishing charge, in which the number of particles and holes is balanced, we show that vortices do not experience any Magnus force. This leads to a soliton decay without snaking, setting it apart from soliton dynamics in non-particle-hole-symmetric fluids, such as BECs. To induce soliton dynamics of the quantum fluid in the BCS limit, we propose to imprint a soliton on the BEC side of the crossover in the presence of a potential barrier. As the next step, we propose to ramp the fluid adiabatically across the crossover into the BCS limit while keeping the barrier potential up. Finally, the barrier potential is ramped to zero, to induce the soliton dynamics. This protocol of initializing the dynamics enables imprinting of the phase pattern with an off-resonant optical pulse, whereas direct phase imprinting in the particlehole-symmetric limit is prohibited. We note that this statement holds only for an exact particle-hole-symmetric case. In experiments such as those in Ref. [27], particle-hole symmetry is only approximately realized. That is, the appropriate effective 


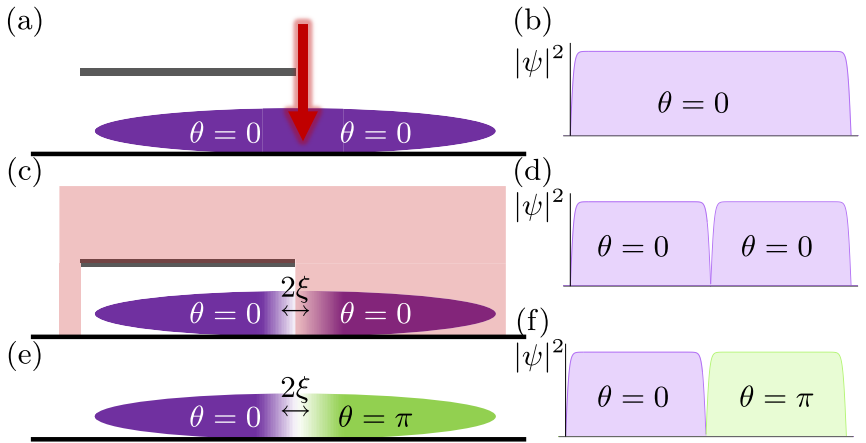

FIG. 1. Schematic representation of (a), (c), and (e) the proposed protocol to imprint a soliton and (b), (d), and (f) the corresponding density profiles $|\psi|^{2}$ and phase distributions $\theta$. (a) A narrow laser sheet is applied to a quantum fluid on the BEC side of the crossover to create a density depletion in the condensate. (b) and (d) The quantum fluid is split into two subsystems with a relative phase of zero. (c) Next a $\pi$ pulse is applied to half of the condensate to create (e) and (f) the phase pattern of a dark soliton. Next the interaction is adiabatically changed across the crossover deep into the BCS side. The narrow laser sheet separating the two subsystems is removed, which triggers the soliton dynamics. Here $\xi$ is the healing length of the condensate.

action is expected to have both $K_{1} \partial_{t}$ and $K_{2} \partial_{t}^{2}$ contributions, as we discuss below. The $K_{1} \partial_{t}$ term allows the phase imprinting as it is the dominant term in the BEC regime. The proposed protocol is displayed in Fig. 1.

\section{SYSTEM}

We consider a low-energy effective models of the form [3]

$$
\begin{aligned}
\mathcal{S}= & \int d^{2} x d t\left(K_{2}\left(\partial_{t} \psi\right)\left(\partial_{t} \bar{\psi}\right)-i K_{1}\left(\partial_{t} \psi\right) \bar{\psi}\right. \\
& -\frac{1}{m} \nabla \bar{\psi} \nabla \psi-\mu|\psi|^{2}+\frac{g}{2}|\psi|^{4}+V_{\mathrm{ext}}|\psi|^{2} \\
& \left.-i \mu_{\mathrm{Q}}\left[\left(\partial_{t} \bar{\psi}\right) \psi-\bar{\psi} \partial_{t} \psi\right]\right),
\end{aligned}
$$

where $K_{1,2}$ are the above-mentioned parameters that determine the time dependence, $\mu$ is the square root of the gap energy, which has the dimensions of a mass term, $g$ is the contact interaction strength, and $V_{\text {ext }}$ is the externally applied potential. A similar effective field theory has been proposed and discussed to model the BEC-BCS crossover in [31-34]. We include a Lagrange multiplier $\mu_{\mathrm{Q}}$ to fix the Klein-Gordon charge (7) (discussed below). By setting $K_{2}=0, K_{1}=1$, and $\mu_{\mathrm{Q}}=0$, we recover the GP equation

$$
i \partial_{t} \psi(\mathbf{x}, t)=\frac{\nabla^{2}}{2 m} \psi(\mathbf{x}, t)+V\left(|\psi|^{2}\right) \psi(\mathbf{x}, t),
$$

where $V\left[|\psi(\mathbf{x}, t)|^{2}\right]=\mu-g|\psi(\mathbf{x}, t)|^{2}+V_{\text {ext }}(\mathbf{x})$. We refer to a condensate described by the GP equation as a GP fluid. This equation is manifestly not particle-hole symmetric under the exchange $\psi \leftrightarrow \bar{\psi}$. On the other hand, particle-hole symmetry is fulfilled in the action (1) by setting $K_{1}=0$ and $K_{2} \neq 0$.
We introduce a dimensionless representation via $\psi=\tilde{\psi} / \xi$, $\nabla=\tilde{\nabla} / \xi, \partial_{t}=c_{s} / \xi \tilde{\partial}_{t}$, and $V=\mu \tilde{V}$, where $\xi$ is the healing length of the fluid and $c_{s}$ the speed of sound. This leads to the modified nonlinear Klein-Gordon (NLKG) equation

$$
\partial_{\tilde{t}}^{2} \tilde{\psi}(\mathbf{x}, t)=\tilde{\nabla}^{2} \tilde{\psi}(\mathbf{x}, t)+\tilde{V}\left(|\tilde{\psi}|^{2}\right) \tilde{\psi}(\mathbf{x}, t)+i \mu_{\mathrm{Q}} \partial_{\tilde{t}} \tilde{\psi}(\mathbf{x}, t) .
$$

We refer to condensates evolving according to the NLKG equation as Klein-Gordon (KG) fluids. In the following we drop the tilde. We trap the fluid using a box potential of the form

$$
V_{\text {ext }}(\mathbf{x})=V_{0}\left\{1+\tanh \left[\left(|\mathbf{x}|-r_{0}\right) / \xi\right]\right\} .
$$

We note that this model is a relativistic BEC [35-37] and a similar equation has been proposed to model cold dark matter [38-40] and relativistic boson stars [41-43].

In the following we show the influence of particle-hole symmetry on the dynamics of topological defects. For the $\mathrm{KG}$ fluid, we introduce the canonical momentum $\Pi(x, t)=$ $\partial_{t} \bar{\psi}(x, t)+i \mu_{\mathrm{Q}} \bar{\psi}(x, t)$ to obtain two coupled first-order partial differential equations

$$
\begin{gathered}
\partial_{t} \psi(\mathbf{x}, t)=\bar{\Pi}(\mathbf{x}, t)+i \mu_{\mathrm{Q}} \psi(\mathbf{x}, t) \\
\partial_{t} \Pi(\mathbf{x}, t)=\nabla^{2} \bar{\psi}(\mathbf{x}, t)+V\left(|\psi|^{2}\right) \bar{\psi}(\mathbf{x}, t)-i \mu_{\mathrm{Q}} \Pi(\mathbf{x}, t) .
\end{gathered}
$$

A crucial feature of a KG fluid is that the particle number $N=\int|\psi(\mathbf{x}, t)|^{2} d x$ is not conserved, in contrast to a GP fluid. Instead, in the KG fluid, the Noether charge

$$
Q=-i \int[\bar{\Pi}(\mathbf{x}, t) \bar{\psi}(\mathbf{x}, t)-\Pi(\mathbf{x}, t) \psi(\mathbf{x}, t)] d^{2} x
$$

is conserved. The Noether charge $Q$ can be thought of as the difference of particles and holes in the system. That is, a zero Noether charge describes the situation with an equal number of particles and holes. An intuitive example for illustrating the Noether charge is a system of interacting bosons in an optical lattice with unit filling. An excitation corresponds to exciting one atom out of the lattice side and leaving behind a hole. Thus, the Noether charge stays unchanged as the same number of particles and hole were created. Another possible excitation is to excite the atom out of the lattice and further removing it from the system, which leaves a hole behind. The system then slightly goes away from unit filling as there is now an imbalance between the number of holes and particles and this corresponds to an effective nonzero Noether charge. Another example can be envisioned in the BCS regime for nonzero temperature. Here a rf knife can be used to remove some of the atoms occupying the Bogoliubov modes, leading to an imbalance between particle and hole excitations.

We apply the Madelung transformation to the field and the canonical momentum, in which the field $\psi$ is written in an amplitude-phase representation

$$
\begin{gathered}
\psi(\mathbf{x}, t)=A(\mathbf{x}, t) \exp [i \theta(\mathbf{x}, t)], \\
\Pi(\mathbf{x}, t)=\left(\frac{\dot{A}(\mathbf{x}, t)}{A(\mathbf{x}, t)}+i\left[\mu_{\mathrm{Q}}-\dot{\theta}(\mathbf{x}, t)\right]\right) \psi(\mathbf{x}, t),
\end{gathered}
$$


and obtain the continuity equation and particle-holesymmetric Euler equation

$$
\begin{gathered}
\partial_{t} \rho_{\mathrm{KG}}+\frac{\mu_{\mathrm{Q}}}{2} \partial_{t} \rho_{\mathrm{S}}=-\nabla\left(\rho_{\mathrm{S}} \mathbf{u}\right), \\
\left(\frac{\rho_{\mathrm{KG}}}{\rho_{\mathrm{S}}}+\frac{\mu_{\mathrm{Q}}}{2}\right) \partial_{t} \mathbf{u}=\mathbf{u} \nabla \mathbf{u}+\frac{\nabla \rho_{\mathrm{S}}}{2 \rho_{0}}-\frac{\nabla}{2}\left(\frac{\square \sqrt{\rho_{\mathrm{S}}}}{\sqrt{\rho_{\mathrm{S}}}}\right),
\end{gathered}
$$

where we introduce the GP density $\rho_{\mathrm{S}}=A^{2}$, the KG density $\rho_{\mathrm{KG}}=A^{2} \partial_{t} \theta$, the velocity $\mathbf{u}=\nabla \theta$, and the box operator $\square=\partial_{t}^{2}-\nabla^{2}$. In this representation, the charge simplifies to $Q=\int \rho_{\mathrm{KG}} d x$. In the particle-hole-symmetric Euler equations there is a prefactor $\rho_{\mathrm{KG}} / \rho_{\mathrm{S}}$ in front of the time derivative of the velocity field $\partial_{t} \mathbf{u}$. This prefactor depends on the charge $Q$. This is a crucial difference to the GP Euler equation where this prefactor is always 1 .

The particle-hole-symmetric Euler equation (11) has two quantum pressure terms. One term is due to the kinetic energy of the condensate and is proportional to $\frac{\nabla^{2} \sqrt{\rho_{\mathrm{S}}}}{\sqrt{\rho_{\mathrm{S}}}}$. It is the zeropoint motion of the condensate and becomes dominant if the condensate has spatial variations on short length scales [44]. The second is proportional to $\frac{\partial_{t}^{2} \sqrt{\rho_{\mathrm{S}}}}{\sqrt{\rho_{\mathrm{S}}}}$ and originates from the second-order time derivative. It only exists for particle-holesymmetric condensates.

We present the local velocity field around a single vortex. Therefore, we transform into the Feshbach-Villars basis, which translates the NLKG to coupled GP equations for the particles and antiparticles, respectively [45]

$$
\begin{aligned}
& \psi=\frac{1}{\sqrt{2}}\left(\psi^{\mathrm{p}}+\psi^{\mathrm{a}}\right), \\
& \Pi=\frac{i}{\sqrt{2}}\left(\psi^{\mathrm{a}}-\psi^{\mathrm{p}}\right) .
\end{aligned}
$$

Next we expand the field around the vortex core position $r_{0}$ with the amplitude $A^{\mathrm{i}}$ and phase $\theta^{\mathrm{i}}$ [see Eqs. (8) and (9)] and propagate the location of the vortex core using the equations of motion and compare the new location with the previous location to obtain the local velocity field (for a detailed discussion and derivation see [46-48]). For the two velocity fields we obtain

$$
\begin{gathered}
v^{\mathrm{a}}=-\frac{(-i, 1)^{\mathrm{T}} \cdot \nabla\left(A^{\mathrm{p}}+A^{\mathrm{a}}\right)+\left(A^{\mathrm{p}}+A^{\mathrm{a}}\right)(1, i)^{\mathrm{T}} \cdot \nabla \theta}{A^{\mathrm{a}}}, \\
v^{\mathrm{p}}=\frac{(-i, 1)^{\mathrm{T}} \cdot \nabla\left(A^{\mathrm{p}}+A^{\mathrm{a}}\right)+\left(A^{\mathrm{p}}+A^{\mathrm{a}}\right)(1, i)^{\mathrm{T}} \cdot \nabla \theta}{A^{\mathrm{p}}},
\end{gathered}
$$

where the spatial plane $(x, y)$ is represented as the complex plane $z=x+i y$. Translating this back into the $(\psi, \Pi)$ basis, we obtain

$$
v^{\psi}=\frac{1}{\sqrt{2}}\left(v^{\mathrm{p}}+v^{\mathrm{a}}\right)=\sqrt{2}\left(1-\frac{A^{\mathrm{a}}}{A^{\mathrm{p}}}\right) v^{\mathrm{p}} .
$$

For $Q \neq 0$ we have $A^{\mathrm{a}} \neq A^{\mathrm{p}}$, which means that we obtain a nonzero velocity field. In this case the velocity is proportional to the velocity obtained for GP fluids [47]. For $Q=0$, we have $A^{\mathrm{p}}=A^{\mathrm{a}}$ and $N^{\mathrm{p}}=N^{\mathrm{a}}$, with $N^{i}$ the total number of particles/antiparticles. For this balanced scenario the local velocity field vanishes precisely as shown in Fig. 2. As pointed out before and as can be seen from Eq. (16), for a finite charge
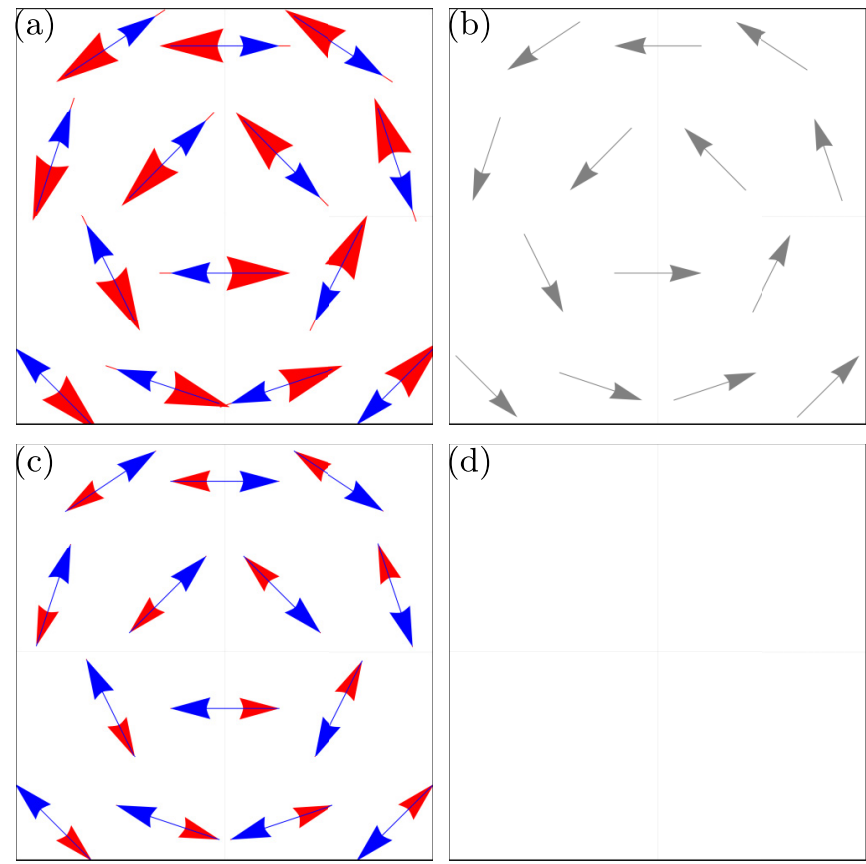

FIG. 2. Schematic sketch of (a) and (c) the local velocity fields of the particles (blue) and antiparticles (red) and (b) and (d) the resulting total local velocity field for the $\psi$ field (gray). (a) An unbalanced mixture of particles and antiparticles with a finite charge $Q$ leads to (b) a nonzero effective velocity field for the $\psi$ field. (c) A balanced mixture of particles and antiparticles with a vanishing charge $Q$ leads to (d) a vanishing effective velocity field for the $\psi$ field.

$Q$ corresponding to an imbalance between particles and antiparticles, the magnitudes of the velocity fields are different [see Fig. 2(a)], which results in a nonzero velocity field for the KG fluid $\psi$ [see Fig. 2(b)]. In contrast, for a balanced mixture the local velocity field magnitudes are the same [see Fig. 2(c)] and due to the opposite direction of the velocity fields the velocity field of the KG fluid vanishes [see Fig. 2(d)].

\section{NUMERICAL RESULTS}

To expand on our analytical predictions and to propose an experimental setup to detect vortex dynamics of KG fluids, we simulate the equations using the pseudospectral method [49] for both the GP and KG fluids. We set the ratio between the chemical potential $\mu$ and the contact interaction $g$ to $\mu / g=$ $10 / \xi^{2}$. In the following we express all length scales in units of $\xi$. Our simulations are discretized in a $256 \times 256$ grid. We choose $r_{0} / \xi=25$, where $r_{0}$ is half of the box size, as defined in Eq. (4), and resolve $\xi$ with three grid points. The phase and density distribution for snapshots in real time are shown for a GP fluid [see Fig. 3(a)] and for a KG fluid with vanishing charge [see Fig. 3(c)]. Circles (KG fluid with $Q=0$ ) and diamonds (GP fluid) in red correspond to a phase winding of +1 , while blue corresponds to -1 [see Fig. 3(b)]. The gray arrows show the flow of time in the figure. It can be seen that for a dipole distance $d_{12}>2 \xi$ in the GP fluid the dipole will start to propel forward perpendicular to the dipole axis and will not annihilate. In contrast, the KG vortex dipoles will move along the dipole axis and annihilate each other, due to the absence 
(a)

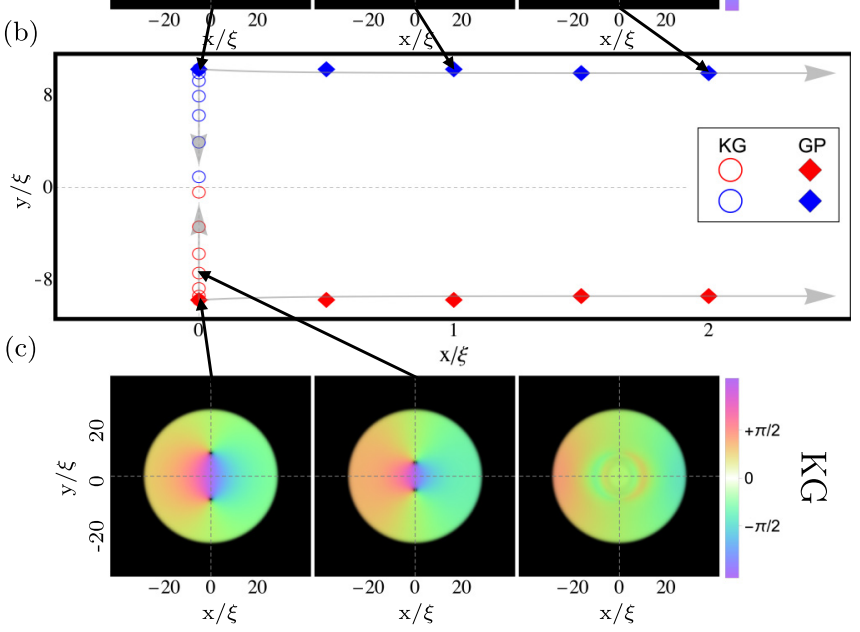

FIG. 3. Dynamics of vortex dipole pairs in a GP and a KG fluid. The phase and the density of (a) the GP fluid and (c) the KG fluid are shown. (b) The symbols display the locations of the vortices and antivortices in red and blue, respectively, of the GP fluid (diamonds) and the KG fluid (circles), and difference times. The snapshots of (a) and (c) are indicated via black arrows. The gray arrows indicate the movement of the vortices in time.

of a velocity field. Related observations of vortex dynamics were reported in Ref. [50]. We note that the particle-hole symmetry is the origin of this qualitatively distinct behavior from GP fluid dynamics. We propose that the data from a future experimental realization of our proposal could be used to numerically fit the ratio of $K_{1}$ and $K_{2}$ for different interaction strengths. This links our proposal to the parameters used in the universal effective action of such systems.

To investigate the influence of the particle-hole symmetry on the soliton dynamics, we initialize the condensate with a modified Thomas-Fermi profile [44], as described in the Supplemental Material [48], for a box potential in Eq. (4) with $V_{0}=10$ and $r_{0}=30$ for the GP $(\mathrm{KG})$ fluid and start with a soliton imprinted in the fluid. We let the condensate relax using the imaginary-time propagation [51] extended to particle-hole-symmetric fluids [48]. For the KG fluids, we set the initial canonical momentum as $\Pi=i \frac{\mu_{\mathrm{Q}}}{2} \psi$ with $\mu_{\mathrm{Q}} \in \mathbb{R}$, resulting in a charge of $Q=\mu_{Q} N$. Furthermore, we add $1 \%$ white noise on the initial condensate density to study the stability of solitons.

The system is propagated in time according to Eqs. (2), (5), and (6). At lowest order, the Higgs mode and the Goldstone mode decouple in a particle-hole-symmetric theory [3]. Within this approximation, this initial state only induces the dynamics of the Goldstone mode. However, this approximation fails in soliton and vortex solutions. For the same parameters $\mu$ and $g$, the healing length is twice as large in the $\mathrm{KG}$ case compared to GP equation due to the difference in the prefactor of the kinetic energy.
In Fig. 4 we present the real-time dynamics of the complex field $\psi$ shaded from white to black corresponding to decreasing amplitude, i.e., black regions denote areas with vanishing $|\psi|$. The phase of the wave function is represented as color. The wave function is normalized for each snapshot such that the maximum value is set to unity to make it easier to compare $\mathrm{GP}$ and $\mathrm{KG}$ results.

In the GP fluid, we observe the established soliton instability in Figs. 4(a)-4(c) [16] and the motion of trapped vortices in Figs. 4(c)-4(e) $[23,47]$. The vortices move towards the edge of the condensate. As they approach the edge, they experience a net force and move along the trap boundary as depicted in Figs. 4(d) and 4(e) [23]. The behavior of the KG fluid with $Q \neq 0$ is similar. As displayed in Figs. 4(f) $-4(\mathrm{j})$, the soliton decays into vortices, which then move around the condensate. Similar to the GP fluid, as the phase rotates in Figs. 4(f)-4(j), the vortices experience a net force leading to their motion along the trap boundary as seen in Figs. 4(i)-4(j).

In contrast, for the $\mathrm{KG}$ fluid with $Q=0$, the soliton decays into vortices that are located along the soliton axis, as shown in Figs. 4(k)-4(m). Similar results have been found in [28]. Moreover, we find that the vortices are not rotating as displayed in Figs. 4(m)-4(o), which is consistent with Eqs. (14) and (16) and Fig. 3. When the vortices reach the trap boundary, they evaporate into the thermal cloud as shown in Figs. 4(n) and 4(o).

\section{CONCLUSION}

In conclusion, we have shown that by measuring the density profile of a two-dimensional condensate after imprinting a soliton in a particle-hole-symmetric superfluid, such as a BCS state of neutral particles, it is possible to test the effective low-energy theoretical description of the system. We have shown analytically and numerically that for particlehole-symmetric superfluids with vanishing Noether charge, the Magnus force is absent. This allows for a dipole pair of vortices to approach each other without transverse motion and to annihilate, reminiscent of a recent observation in Ref. [50]. Another consequence of the vanishing Magnus force is that a soliton does not bend as it decays into vortices. Probing these effects experimentally will reveal how well particle-hole symmetry is realized in the dynamics of superfluids or whether the non-particle-hole-symmetric term, the first-order derivative in time, is the dominant contribution in the effective theory. This is crucial in understanding the notion of turbulence in particle-hole-symmetric fluids such as superconductors. Our work reveals that turbulence in a BCS superconductor and its scaling laws might deviate from Kolmogorov scaling laws [52], which apply to classical systems as well to GP fluids. We note that our predictions could be experimentally confirmed using refined experimental technique, such as in situ observations of two-dimensional Fermi liquids when probing the BEC-BCS crossover in neutral atoms $[53,54]$ or the wellcontrolled imprinting of vortex dipole pairs [50].

\section{ACKNOWLEDGMENTS}

We thank Guido Homann and Antonio Muñoz Mateo for fruitful discussions. This work was supported by the Deutsche Forschungsgemeinschaft in the framework of SFB 

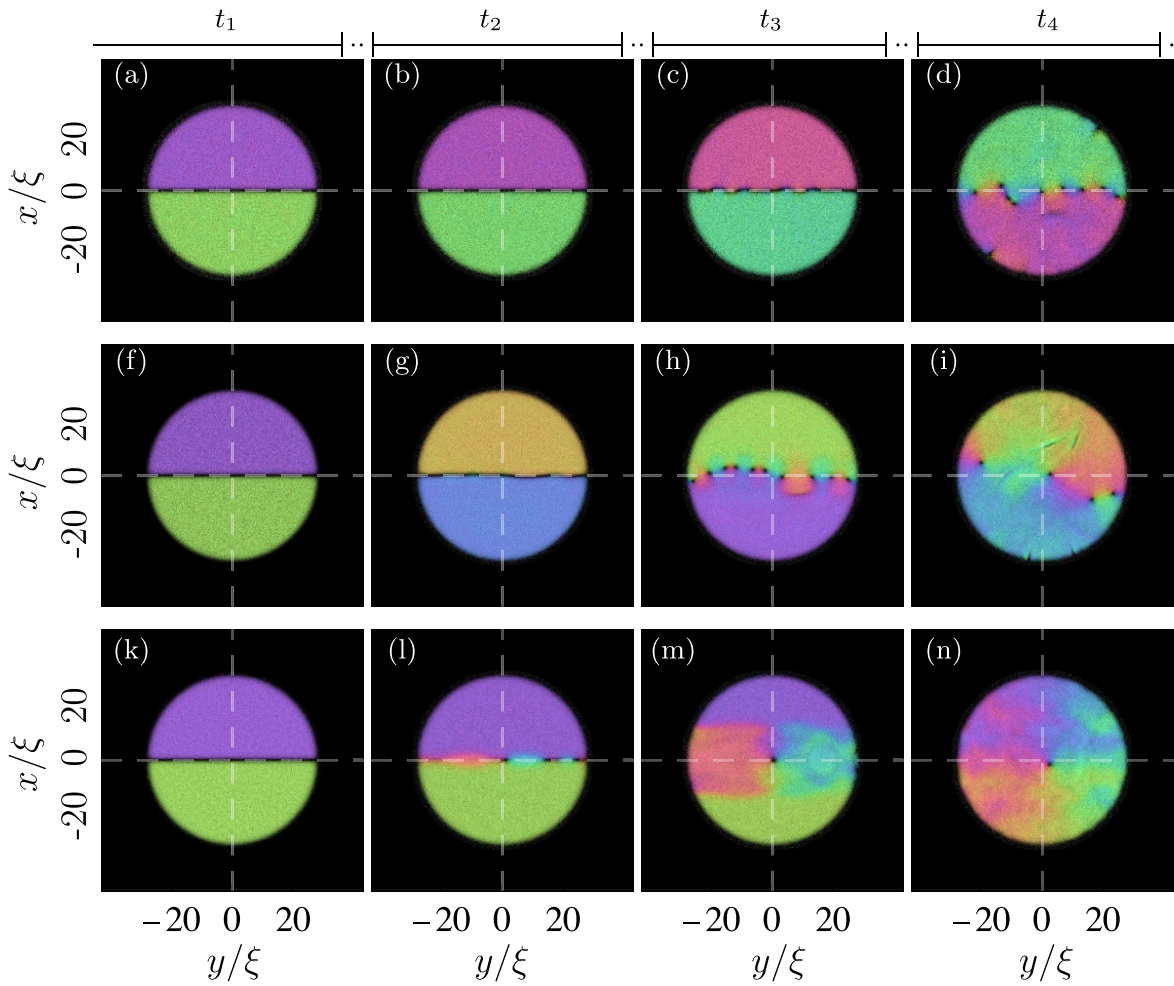

$\begin{array}{lll}-20 & 0 & 20\end{array}$

$y / \xi$
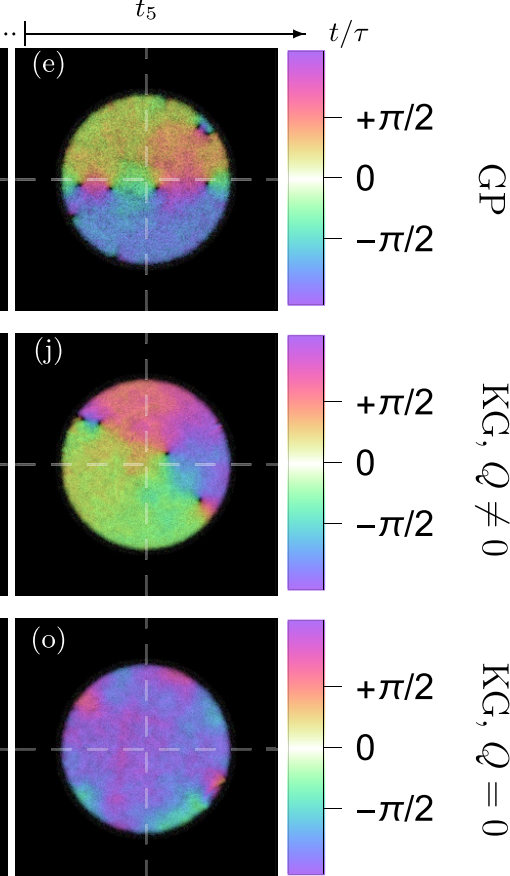

\section{$\sqrt{2}$
0
0}

FIG. 4. Overview of the soliton instability for the three distinct cases corresponding to (a)-(e) the GP results, (f)-(j) the KG results with $Q \neq 0$, and (k)-(o) the $\mathrm{KG}$ results with $Q=0$. For a better comparison between the GP and $\mathrm{KG}$ results the $\psi$ field density is normalized such that the maximum value is set to unity in each snapshot. The shading of the plots ranging from black to white visualizes the magnitude of the field $|\psi|$, while the colormap indicates the phase. (a), (f), and (k) Initial soliton seeded with white noise. (b), (g), and (l) Soliton bending. (c), (h), and (m) Vortices appearing after the soliton decay. The long-time dynamics of the vortices inside the trap are presented in (d), (i), and (n) and in (e), (j), and (o). White dashed lines indicate the soliton axis and the perpendicular axis. The spatial length is expressed in terms of the healing length $\xi$. A movie showing the dynamics is presented in the Supplemental Material [48].

925, Project No. 170620586, and the Cluster of Excellence "Advanced Imaging of Matter" (EXC 2056), Project No.
390715994. J.S. acknowledges support from the German Academic Scholarship Foundation.
[1] S. Weinberg, The Quantum Theory of Fields (Cambridge University Press, Cambridge, 2013), Vol. 2.

[2] S. Sachdev, Quantum Phase Transitions, 2nd ed. (Cambridge University Press, Cambridge, 2011).

[3] D. Pekker and C. Varma, Amplitude/Higgs modes in condensed matter physics, Annu. Rev. Condens. Matter Phys. 6, 269 (2015).

[4] C. M. Varma, Higgs boson in superconductors, J. Low Temp. Phys. 126, 901 (2002).

[5] G. Homann, J. G. Cosme, and L. Mathey, Higgs time crystal in a high- $T_{c}$ superconductor, Phys. Rev. Res. 2, 043214 (2020).

[6] G. Homann, J. G. Cosme, J. Okamoto, and L. Mathey, Higgs mode mediated enhancement of interlayer transport in high- $T_{c}$ cuprate superconductors, Phys. Rev. B 103, 224503 (2021).

[7] Z. Dai and P. A. Lee, Photo-induced superconducting-like response in strongly correlated systems, Phys. Rev. B 104, 054512 (2021).

[8] Z. Dai and P. A. Lee, Superconducting-like response in driven systems near the Mott transition, arXiv:2106.08354.

[9] E. Altman and A. Auerbach, Oscillating Superfluidity of Bosons in Optical Lattices, Phys. Rev. Lett. 89, 250404 (2002).
[10] L. Pollet and N. Prokof'ev, Higgs Mode in a Two-Dimensional Superfluid, Phys. Rev. Lett. 109, 010401 (2012).

[11] J. Léonard, A. Morales, P. Zupancic, T. Donner, and T. Esslinger, Monitoring and manipulating Higgs and Goldstone modes in a supersolid quantum gas, Science 358, 1415 (2017).

[12] M. Endres, T. Fukuhara, D. Pekker, M. Cheneau, P. Schauss, C. Gross, E. Demler, S. Kuhr, and I. Bloch, The 'Higgs' amplitude mode at the two-dimensional superfluid/Mott insulator transition, Nature (London) 487, 454 (2012).

[13] A. Behrle, T. Harrison, J. Kombe, K. Gao, M. Link, J. S. Bernier, C. Kollath, and M. Köhl, Higgs mode in a strongly interacting fermionic superfluid, Nat. Phys. 14, 781 (2018).

[14] V. E. Zakharov and A. M. Rubenchik, Instability of waveguides and solitons in nonlinear media, Sov. Phys._JETP 38, 494 (1974).

[15] C. A. Jones, S. J. Putterman, and P. H. Roberts, Motions in a Bose condensate. V. Stability of solitary wave solutions of non-linear Schrodinger equations in two and three dimensions, J. Phys. A: Math. Gen. 19, 2991 (1986).

[16] J. Brand and W. P. Reinhardt, Solitonic vortices and the fundamental modes of the "snake instability": Possibility of 
observation in the gaseous Bose-Einstein condensate, Phys. Rev. A 65, 043612 (2002).

[17] A. Muñoz Mateo and J. Brand, Chladni Solitons and the Onset of the Snaking Instability for Dark Solitons in Confined Superfluids, Phys. Rev. Lett. 113, 255302 (2014).

[18] P. G. Kevrekidis, I. Danaila, J.-G. Caputo, and R. CarreteroGonzález, Planar and radial kinks in nonlinear Klein-Gordon models: Existence, stability, and dynamics, Phys. Rev. E 98, 052217 (2018).

[19] N.-E. Guenther, P. Massignan, and A. L. Fetter, Quantized superfluid vortex dynamics on cylindrical surfaces and planar annuli, Phys. Rev. A 96, 063608 (2017).

[20] L. A. Toikka and J. Brand, Asymptotically solvable model for a solitonic vortex in a compressible superfluid, New J. Phys. 19, 023029 (2017).

[21] B. Jackson, J. F. McCann, and C. S. Adams, Vortex line and ring dynamics in trapped Bose-Einstein condensates, Phys. Rev. A 61, 013604 (1999).

[22] S. A. McGee and M. J. Holland, Rotational dynamics of vortices in confined Bose-Einstein condensates, Phys. Rev. A 63, 043608 (2001).

[23] D. E. Sheehy and L. Radzihovsky, Vortices in spatially inhomogeneous superfluids, Phys. Rev. A 70, 063620 (2004).

[24] J. Denschlag, J. E. Simsarian, D. L. Feder, C. W. Clark, L. A. Collins, J. Cubizolles, L. Deng, E. W. Hagley, K. Helmerson, W. P. Reinhardt, S. L. Rolston, B. I. Schneider, and W. D. Phillips, Generating solitons by phase engineering of a BoseEinstein condensate, Science 287, 97 (2000).

[25] S. Burger, K. Bongs, S. Dettmer, W. Ertmer, K. Sengstock, A. Sanpera, G. V. Shlyapnikov, and M. Lewenstein, Dark Solitons in Bose-Einstein Condensates, Phys. Rev. Lett. 83, 5198 (1999).

[26] M. W. Zwierlein, J. R. Abo-Shaeer, A. Schirotzek, C. H. Schunck, and W. Ketterle, Vortices and superfluidity in a strongly interacting Fermi gas, Nature (London) 435, 1047 (2005).

[27] M. J. H. Ku, W. Ji, B. Mukherjee, E. Guardado-Sanchez, L. W. Cheuk, T. Yefsah, and M. W. Zwierlein, Motion of a Solitonic Vortex in the BEC-BCS Crossover, Phys. Rev. Lett. 113, 065301 (2014).

[28] W. Van Alphen, H. Takeuchi, and J. Tempere, Crossover between snake instability and Josephson instability of dark solitons in superfluid fermi gases, Phys. Rev. A 100, 023628 (2019).

[29] J. Bardeen and M. J. Stephen, Theory of the motion of vortices in superconductors, Phys. Rev. 140, A1197 (1965).

[30] P. Ao and D. J. Thouless, Berry's Phase and the Magnus Force for a Vortex Line in a Superconductor, Phys. Rev. Lett. 70, 2158 (1993).

[31] S. N. Klimin, J. Tempere, and J. T. Devreese, Finite-temperature effective field theory for dark solitons in superfluid Fermi gases, Phys. Rev. A 90, 053613 (2014).

[32] S. N. Klimin, J. Tempere, G. Lombardi, and J. T. Devreese, Finite temperature effective field theory and two-band superfluidity in Fermi gases, Eur. Phys. J. B 88, 122 (2015).

[33] G. Lombardi, W. Van Alphen, S. N. Klimin, and J. Tempere, Soliton-core filling in superfluid Fermi gases with spin imbalance, Phys. Rev. A 93, 013614 (2016).

[34] W. Van Alphen, G. Lombardi, S. N. Klimin, and J. Tempere, Dark soliton collisions in superfluid Fermi gases, New J. Phys. 20, 053052 (2018).
[35] S. Fagnocchi, S. Finazzi, S. Liberati, M. Kormos, and A. Trombettoni, Relativistic Bose-Einstein condensates: A new system for analogue models of gravity, New J. Phys. 12, 095012 (2010).

[36] H. E. Haber and H. A. Weldon, Thermodynamics of an Ultrarelativistic Ideal Bose Gas, Phys. Rev. Lett. 46, 1497 (1981).

[37] M. Grether, M. de Llano, and G. A. Baker, Bose-Einstein Condensation in the Relativistic Ideal Bose Gas, Phys. Rev. Lett. 99, 200406 (2007).

[38] K. Huang, H.-B. Low, and R.-S. Tung, Scalar field cosmology II: Superfluidity, quantum turbulence, and inflation, Int. J. Mod. Phys. A 27, 1250154 (2012).

[39] J. Magaña, T. Matos, A. Suárez, and F. J. SánchezSalcedo, Structure formation with scalar field dark matter: The field approach, J. Cosmol. Astropart. Phys. 2012, 003 (2012).

[40] C. Xiong, M. R. Good, Y. Guo, X. Liu, and K. Huang, Relativistic superfluidity and vorticity from the nonlinear Klein-Gordon equation, Phys. Rev. D 90, 125019 (2014).

[41] M. Colpi, S. L. Shapiro, and I. Wasserman, Boson Stars: Gravitational Equilibria of Self-Interacting Scalar Fields, Phys. Rev. Lett. 57, 2485 (1986).

[42] P.-H. Chavanis and T. Harko, Bose-Einstein condensate general relativistic stars, Phys. Rev. D 86, 064011 (2012).

[43] A. Suárez and P.-H. Chavanis, Hydrodynamic representation of the Klein-Gordon-Einstein equations in the weak field limit: General formalism and perturbations analysis, Phys. Rev. D 92, 023510 (2015).

[44] C. J. Pethick and H. Smith, Bose-Einstein Condensation in Dilute Gases, 2nd ed. (Cambridge University Press, Cambridge, 2008).

[45] H. Feshbach and F. Villars, Elementary relativistic wave mechanics of spin 0 and spin 1/2 particles, Rev. Mod. Phys. 30, 24 (1958).

[46] O. Törnkvist and E. Schröder, Vortex Dynamics in Dissipative Systems, Phys. Rev. Lett. 78, 1908 (1997).

[47] A. J. Groszek, D. M. Paganin, K. Helmerson, and T. P. Simula, Motion of vortices in inhomogeneous Bose-Einstein condensates, Phys. Rev. A 97, 023617 (2018).

[48] See Supplemental Material at http://link.aps.org/supplemental/ 10.1103/PhysRevResearch.3.043109 for a movie.

[49] W. Bao, D. Jaksch, and P. A. Markowich, Numerical solution of the Gross-Pitaevskii equation for Bose-Einstein condensation, J. Comput. Phys. 187, 318 (2003).

[50] W. J. Kwon, G. D. Pace, K. Xhani, L. Galantucci, A. M. Falconi, M. Inguscio, F. Scazza, and G. Roati, Sound emission and annihilations in a programmable quantum vortex collider, arXiv:2105.15180.

[51] C. Barenghi and N. G. Parker, A Primer on Quantum Fluids (Springer, Cham, 2016).

[52] A. Kolmogorov, The local structure of turbulence in incompressible viscous fluid for very large Reynolds numbers, Dokl. Akad. Nauk SSSR 30, 301 (1941).

[53] K. Hueck, N. Luick, L. Sobirey, J. Siegl, T. Lompe, and H. Moritz, Two-Dimensional Homogeneous Fermi Gases, Phys. Rev. Lett. 120, 060402 (2018).

[54] L. Sobirey, N. Luick, M. Bohlen, H. Biss, H. Moritz, and T. Lompe, Observation of superfluidity in a strongly correlated two-dimensional Fermi gas, Science 372, 844 (2021). 